\title{
Brucelosis en Chile: Descripción de una serie de 13 casos
}

\author{
Roberto Olivares, Pamela Vidal, Camila Sotomayor, Mackarenna Norambuena, \\ Mario Luppi, Francisco Silva, Marcela Cifuentes
}

\section{Brucellosis in Chile: Description of a series of 13 cases}

Introduction: Brucellosis is a zoonosis caused by Brucella spp. It may be acquired by consuming unpasteurized dairy products. Brucellosis has a low incidence in Chile, thus, we have a scarce data. Aim: To report and to characterize the first series of clinical cases of adult patients diagnosed with brucellosis in Chile. Methods: We describe a series of 13 clinical cases in patients diagnosed between 2000 and 2016 in three different centers in the Metropolitan Region, Chile. A retrospective analysis was performed on clinical presentation, laboratory, antibiotic treatment, morbidity and mortality. Results: The mean age was 50 years old. Eight cases had a record of consumption of unpasteurized dairy products. The most frequently reported complaints were fever. The most frequent focal point involved was the spine. Only one patient had a positive blood culture, while the diagnosis was made using serological techniques in the other part of the group. The most indicated antibiotic regimens were doxycycline-rifampicin and doxycycline-gentamicin. The hospital stay was 20 days approximately as an average. Clinical cure was achieved in all cases. Conclusions: Brucellosis is an infrequent zoonosis in Chile, and it produces a nonspecific clinical picture, so it is necessary to have high suspicion to make the diagnosis based in the antecedent of consumption of unpasteurized dairy or raw meat.

Key words: Brucella; brucelosis; zoonosis.

Palabras clave: Brucella; brucelosis; zoonosis.

\section{Introducción}

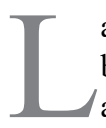
a brucelosis es una zoonosis causada por una bacteria del género Brucella, y es una de las más ampliamente distribuidas en el mundo ${ }^{1}$. Se estima que provoca más de 500.000 casos nuevos al año. Se transmite a los seres humanos mediante el consumo de productos lácteos no pasteurizados, carne infectada de ganado doméstico o contacto con fluidos de animales infectados, vivos o muertos ${ }^{2}$. Se considera controlada en la mayoría de los países desarrollados, pero nuevos focos de reemergencia han aparecido durante los últimos años, modificando su epidemiología ${ }^{3}$. Se considera endémica en regiones de Asia, África, y Sudamérica, configurando un problema importante de salud pública debido al impacto en morbilidad y pérdidas económicas, que esta zoonosis provoca $^{4}$, tanto en humanos como en animales. En Chile, la incidencia promedio sería de 0,55 casos por $100 \mathrm{mil}$ habitantes $^{2}$, pero se considera subestimada, siendo 10 a 25 veces más alta que la reportada a nivel nacional ${ }^{5}$.

La brucelosis en Chile es una entidad clínica de baja ocurrencia, y los datos nacionales acerca de esta enfermedad en cuanto a forma de presentación, diagnóstico y tratamiento son escasos.

El objetivo de este estudio es reportar por primera vez y caracterizar una serie de casos clínicos de pacientes adultos con diagnóstico de brucelosis en Chile.

\section{Material y Métodos}

Se trata de un estudio descriptivo, retrospectivo, multicéntrico, con un total de 13 casos ingresados en el Hospital Clínico de la Universidad de Chile, Hospital Clínico San Borja Arriarán y Clínica Dávila (todos en Santiago), incluidos en el estudio. Se analizaron fichas clínicas en papel y electrónica de pacientes con diagnóstico de brucelosis en el período comprendido entre junio de 2000 y septiembre de 2016.

Las variables a considerar fueron: sexo, edad, factores de riesgo, hallazgos clínicos al ingreso y durante la estadía hospitalaria, hallazgos de laboratorio, método diagnóstico, tratamiento indicado, duración de la estadía hospitalaria y morbi-mortalidad.

Se estimó el período de tiempo entre el inicio de la sintomatología y la hospitalización en cada caso, a partir de la anamnesis registrada en la ficha clínica. De acuerdo a esto, se catalogaron como brucelosis aguda aquellos casos en que el período sintomático fue menor a dos meses, como brucelosis subaguda aquellos en que la evolución de los síntomas se encontró entre dos y 12 meses y brucelosis crónica en aquellos pacientes con manifestaciones clínicas de más de 12 meses de duración ${ }^{6}$.

El diagnóstico de brucelosis fue realizado en base al aislamiento de Brucella spp en sangre, o la presencia de cuadro clínico sugerente, asociado a la detección de
Hospital Clínico de la Universidad de Chile. Sección Infectología (ROC, MLN) Laboratorio de Microbiología (FSO).

Universidad de Chile, Facultad de Medicina.

Interna de VII Medicina (PVL, CSL, MNG)

Hospital Clínico San Borja Arriarán.

Laboratorio de Microbiología. (MCD).

Los autores declaran no tener conflictos de interés.

Fuente de financiamiento: No hubo.

Recibido: 10 de enero de 2017 Aceptado: 30 de mayo de 2017

Correspondencia a: Roberto Olivares Castillo rocroz@gmail.com 
anticuerpos específicos en títulos significativos $(\geq 1: 80)$ en la prueba de aglutinación en suero de Wright, de acuerdo al valor de corte aceptado para diagnóstico de brucelosis en países de baja endemia ${ }^{7}$, o detección de anticuerpos específicos mediante la técnica de enzimo-inmunoensayo, (ELISA).

Una respuesta adecuada a terapia anti brucelosis también se aceptó como criterio diagnóstico en aquellos pacientes con cultivos negativos o con serología en títulos no significativos ${ }^{8}$.

Respecto a exámenes de laboratorio generales, se consideraron alterados si se obtenían valores fuera del valor normal de referencia del laboratorio del centro hospitalario respectivo.

Se definió como infección focal la presencia de síntomas o signos de infección en un sitio anatómico durante siete o más días. Se definió como compromiso óseo y/o articular a la presencia de dolor de carácter inflamatorio, asociado a alteración radiológica y/o aumento de captación en la cintigrafía en una estructura ósea o articulación profunda. El compromiso hepático fue definido como la presencia de absceso hepático o aumento en cinco veces o más respecto al límite del valor normal de transaminasas GOT o GPT o aumento en tres veces o más respecto al límite del valor normal de fosfatasa alcalina o transaminasa GGT, asociado a ictericia o dolor en el cuadrante superior derecho del abdomen. Se consideró como compromiso neurológico cualquier síntoma o signo neurológico no atribuible a otras causas, asociado al aislamiento simultáneo de Brucella spp en líquido cefalorraquídeo. Dentro de las complicaciones hematológicas sólo se incluyeron alteraciones de laboratorio asociadas a manifestaciones clínicas, excluyéndose las asintomáticas u oligo-sintomáticas ${ }^{9}$.

Se consideraron como tratamiento los esquemas de antimicrobianos indicados tras el diagnóstico de brucelosis, durante la estadía hospitalaria y al alta. No se realizó seguimiento sistemático tras el alta hospitalaria.

Este trabajo fue aprobado íntegramente para su realización por el Comité de Ética del Hospital Clínico de la Universidad de Chile.

Tabla 1. Descripción demográfica de los pacientes con brucelosis incluidos en el estudio

\begin{tabular}{|c|c|c|c|c|c|}
\hline Sexo & $\mathrm{n}$ casos & $\%$ & Centro asistencial & n casos & $\%$ \\
\hline Mujer & 5 & 38,5 & Clínica Dávila & 1 & 9,76 \\
\hline Hombre & 8 & 61,5 & $\mathrm{HCSBA}^{1}$ & 2 & 4,15 \\
\hline \multirow[t]{2}{*}{ Total } & 13 & 100 & $\mathrm{HCUCh}^{2}$ & 10 & 7,7 \\
\hline & & & Total & 13 & 100 \\
\hline
\end{tabular}

${ }^{1}$ HCSBA: Hospital Clínico San Borja Arriarán. ${ }^{2} \mathrm{HCUCh}$ : Hospital Clínico de la Universidad de Chile.

\section{Resultados}

De los 13 pacientes, ocho eran hombres y cinco eran mujeres. El rango de edad estuvo entre 24 y 76 años, con un promedio de $50 \pm 17$ años. Del total, 10 casos ingresaron al Hospital Clínico de la Universidad de Chile, dos casos al Hospital Clínico San Borja Arriarán y un caso ingresó a Clínica Dávila (Tabla 1).

De los 13 casos, ocho tenían el antecedente de consumo de lácteos no pasteurizados, dos el antecedente de consumo de carne mal cocida y en tres casos no se indagó al respecto durante la estadía hospitalaria.

El motivo de consulta más frecuente fue sensación febril. Otros, fueron compromiso del estado general, disnea, mialgias, artralgias, odinofagia, dolor abdominal y dolor lumbar.

El tiempo de evolución previo a la hospitalización fue, en promedio, 52 días, variando desde 3 hasta 210 días. De acuerdo a esto, se categorizaron siete casos como brucelosis aguda, y seis casos como brucelosis subaguda. No se pesquisó caso alguno de brucelosis crónica.

Del grupo de brucelosis aguda, seis eran hombres y dos eran mujeres, y la edad promedio fue de $40 \pm 14$ años.

El grupo de brucelosis subaguda fue conformado por tres hombres y tres mujeres, y la edad promedio fue de 62 \pm 13 años. Cinco de los seis casos que se categorizaron como brucelosis subaguda tuvieron compromiso focal.

Los síntomas y signos más frecuentes se describen en la Tabla 2, destacando sensación febril (10 casos), compromiso del estado general (6 casos), mialgias y artralgias (4 casos). Cabe destacar que en tres casos no hubo hallazgos en el examen físico, y en cuatro casos la fiebre fue la única alteración encontrada.

Cuatro pacientes tuvieron infección focal, de los que tres correspondieron a compromiso de columna vertebral (espondilodiscitis) y un caso a compromiso hepático (microabscesos hepáticos). Todos estos pacientes tuvieron una presentación subaguda de la enfermedad. No se encontraron hallazgos concordantes con compromiso neurológico ni hematológico en esta serie.

Respecto a las alteraciones de laboratorio, las más frecuentes fueron niveles elevados de VHS, (11 casos) y proteína $\mathrm{C}$ reactiva (PCR), (8 casos), leucocitosis (7 casos) y anemia (6 casos). No se encontraron alteraciones del perfil hepático en los casos (Tabla 3 ).

Se obtuvo prueba de aglutinación en suero de Wright positiva en 12 casos, con títulos de anticuerpos iniciales registrados en 11 de ellos, los que variaron entre 1:80 y 1:5120. Un tercio de los títulos se encontraba en 1:320 al momento del diagnóstico. (Tabla 4). Se obtuvo serología positiva por ELISA para IgG en un caso, en títulos de 22,6 U. En un caso se obtuvo prueba de aglutinación en suero de Wright con títulos de anticuerpos en 1:40, lo que no se considera significativo, pero hubo respuesta favorable a la terapia antimicrobiana. 
Se tomaron hemocultivos periféricos en 10 casos, pero sólo en uno de ellos resultó positivo, aislándose $B$. melitensis.

En cuanto a las terapias indicadas tras el diagnóstico de brucelosis, hubo registro en 12 casos. En la mayoría de ellos se indicó esquemas asociados que incluían: gentamicina endovenosa (160 a $480 \mathrm{mg}$ fraccionados en dos a tres administraciones), rifampicina oral (300 a $900 \mathrm{mg}$ al día, en una o dos tomas), doxiciclina oral (100 mg cada $12 \mathrm{~h}$ ) y cotrimoxazol forte oral (160 mg/800 mg al día). Durante la estadía hospitalaria, los esquemas indicados con mayor frecuencia fueron doxiciclina-gentamicina, y doxiciclina-rifampicina. Otras fueron rifampicina-gentamicina y doxiciclina en monoterapia. Durante la hospitalización se indicó terapia parenteral en ocho casos, y en

\begin{tabular}{|c|c|c|}
\hline & $\mathbf{n}$ & $\%$ \\
\hline \multicolumn{3}{|l|}{ Síntomas } \\
\hline Artralgias & 4 & 30,8 \\
\hline Baja de peso & 3 & 23,1 \\
\hline Calofríos & 3 & 23,1 \\
\hline Cefalea & 3 & 23,1 \\
\hline Compromiso del estado general & 6 & 46,2 \\
\hline Debilidad en extremidades inferiores & 1 & 7,7 \\
\hline Diaforesis & 2 & 15,4 \\
\hline Diarrea & 1 & 7,7 \\
\hline Disnea & 1 & 7,7 \\
\hline Dolor abdominal & 2 & 15,4 \\
\hline Dolor de extremidades inferiores & 1 & 7,7 \\
\hline Lumbalgia & 3 & 23,1 \\
\hline Mialgias & 4 & 30,8 \\
\hline Náuseas / vómitos & 3 & 23,1 \\
\hline Odinofagia & 1 & 7,7 \\
\hline Orina oscura & 2 & 15,4 \\
\hline Otalgia & 1 & 7,7 \\
\hline Palpitaciones & 1 & 7,7 \\
\hline Rinorrea & 1 & 7,7 \\
\hline Sensación febril & 10 & 76,9 \\
\hline Tos & 3 & 23,1 \\
\hline \multicolumn{3}{|l|}{ Hallazgos al examen físico } \\
\hline Adenopatías & 1 & 7,7 \\
\hline Artritis periférica & 1 & 7,7 \\
\hline Fiebre $^{1}$ & 7 & 53,8 \\
\hline Hepatomegalia & 1 & 7,7 \\
\hline Masa palpable & 1 & 7,7 \\
\hline Palidez & 1 & 7,7 \\
\hline Paraparesia proximal & 1 & 7,7 \\
\hline Polipnea & 1 & 7,7 \\
\hline Sensibilidad abdominal & 2 & 15,4 \\
\hline Sensibilidad en la palpación de la fosa lumbar & 2 & 15,4 \\
\hline
\end{tabular}

uno de ellos se realizó cambio de esquema antimicrobiano. Al alta, el esquema más indicado fue doxiciclina-rifampicina. También se indicó doxiciclina en monoterapia, y doxiciclina-cotrimoxazol (Tabla 5). Todos los pacientes con compromiso de columna fueron tratados con doxiciclina-gentamicina durante la hospitalización y luego con doxiciclina-rifampicina.

La duración del tratamiento antimicrobiano parenteral tuvo una media de 11 días, mientras que la terapia antimicrobiana oral se indicó por 57 días, en promedio. Los pacientes completaron una media de 59 días de antibioterapia en total. En los casos que se presentaron con compromiso de columna vertebral se completaron, en promedio, 118 días.

La estadía hospitalaria fue de 20,3 \pm 19,5 días en promedio,

Tabla 3. Brucelosis en 13 adultos. Hallazgos en el laboratorio general

\begin{tabular}{|c|c|c|}
\hline & $\begin{array}{l}\text { Laboratorio no específico } \\
\text { n casos }\end{array}$ & $\%$ \\
\hline Anemia $^{1}$ & 6 & 46,2 \\
\hline Leucocitosis² & 7 & 53,8 \\
\hline Leucopenia $^{3}$ & 2 & 15,4 \\
\hline Trombocitopenia ${ }^{4}$ & 2 & 15,4 \\
\hline$P C R>10 \mathrm{mg} / \mathrm{l}$ & 8 & 61,5 \\
\hline $\mathrm{VHS}>15 \mathrm{~mm} / \mathrm{h}$ & 11 & 84,6 \\
\hline $\mathrm{LDH}^{7}>618$ & 4 & 30,8 \\
\hline Hematuria $^{8}$ & 2 & 15,4 \\
\hline Leucocituria9 $^{9}$ & 1 & 7,7 \\
\hline
\end{tabular}

${ }^{1}$ Anemia: en mujeres hemoglobina $<12 \mathrm{~g} / \mathrm{dL}$; en hombres hemoglobina $<13,5$ $\mathrm{g} / \mathrm{dL}$. ${ }^{2}$ Leucocitosis: leucocitos $>10.000 / \mathrm{mm}^{3}$. ${ }^{3}$ Leucopenia: leucocitos $<4.000 /$ $\mathrm{mm}^{3}$. ${ }^{4}$ Trombocitopenia: plaquetas $<150.000 / \mathrm{mm}^{3}$. ${ }^{5} \mathrm{LDH}$ : lactato deshidrogenasa. ${ }^{6}$ Hematuria: > 3 glóbulos rojos/campo en hombres; $>5$ glóbulos rojos/ campo en mujeres. ${ }^{7}$ Leucocituria: $>5$ células/campo.

Tabla 4. Títulos obtenidos en prueba de seroaglutinación de Wright en 12 adultos

\begin{tabular}{|ccc|}
\hline Títulos & $\begin{array}{c}\text { Prueba de seroaglutinación de Wright } \\
\mathbf{n}\end{array}$ & $\%$ \\
$1: 40$ & 1 & 8,3 \\
$1: 80$ & 3 & 25,0 \\
$1: 160$ & 0 & 0,0 \\
$1: 320$ & 4 & 33,3 \\
$1: 640$ & 2 & 16,7 \\
$1: 2560$ & 1 & 8,3 \\
$1: 5120$ & 1 & 8,3 \\
\hline Total & 12 & 100 \\
\hline
\end{tabular}

Tabla 5. Terapia antimicrobiana indicada para el manejo de brucelosis en 13 adultos

\begin{tabular}{|c|c|c|c|c|c|}
\hline Vía de administración & $\mathbf{n}$ & Promedio tratamiento (días) & Esquema antimicrobiano & $\mathbf{n}$ & Promedio tratamiento (días) \\
\hline Parenteral & 7 & 11 & $\begin{array}{l}\text { Doxiciclina + gentamicina } \\
\text { Gentamicina }+ \text { rifampicina }\end{array}$ & $\begin{array}{l}6 \\
1\end{array}$ & $\begin{array}{l}10,5 \\
14\end{array}$ \\
\hline Oral & 12 & 57,3 & $\begin{array}{c}\text { Doxiciclina + rifampicina } \\
\text { Doxiciclina + cotrimoxazol } \\
\text { Doxiciclina }\end{array}$ & $\begin{array}{l}9 \\
1 \\
2\end{array}$ & $\begin{array}{l}66,6 \\
35 \\
26,5\end{array}$ \\
\hline
\end{tabular}


variando entre los 2 y 70 días. En todos los casos se logró la curación clínica. Un caso fue reingresado en dos ocasiones por reaparición de sintomatología tras el alta. No se registraron fallecimientos.

\section{Discusión}

La brucelosis es una zoonosis infrecuente en nuestro medio. Hasta ahora, en el medio nacional se han publicado datos en relación a la epidemiología nacional ${ }^{2}$, un estudio de diagnóstico con nuevas técnicas serológicas ${ }^{5}$ y sólo un reporte de caso clínico en un niño de 11 años con brucelosis ${ }^{10}$.

Este corresponde al primer reporte de una serie clínica de pacientes adultos con brucelosis, abarcando un período de 16 años.

La magnitud e impacto de esta enfermedad en medicina humana en Chile es poco conocida y probablemente subdiagnosticada ${ }^{10}$, aun cuando se trata de una enfermedad de notificación obligatoria según el decreto número 158 del Ministerio de Salud. De ahí la importancia de esta publicación, que aporta datos sobre la forma de adquisición, presentación clínica, diagnóstico y tratamiento de esta enfermedad infecciosa en nuestro medio.

En relación al cuadro clínico, nuestros pacientes presentaron un cuadro inespecífico, fundamentalmente caracterizado por sensación febril, compromiso del estado general y mialgias, que es lo habitualmente reportado en la literatura médica ${ }^{11}$. Predominaron los casos agudos y subagudos, concentrándose el compromiso focal en los casos de más larga evolución y siendo el compromiso de columna el más frecuente. Llama la atención además que los casos subagudos con focalización se concentraron en los pacientes de mayor edad.

Los hallazgos en el examen físico también fueron variados e inespecíficos, predominando la fiebre en 54\%, por lo que el antecedente epidemiológico más importante pasa a ser aquí el del consumo de productos lácteos no pasteurizados y el de carne mal cocida, que en esta serie está presente en 10 de los 13 pacientes.

En el laboratorio general predominó el aumento de parámetros inflamatorios, con leucocitosis en el hemograma, alza de VHS y aumento de PCR. Destacan también las manifestaciones hematológicas, entre las que se encuentra la anemia, que se presentó en $46 \%$.

Para el diagnóstico definitivo se utilizaron las técnicas de laboratorio recomendadas por la literatura científica. Sólo uno de los 13 pacientes tuvo un hemocultivo positivo para $B$. melitensis. Para el resto, el diagnóstico se realizó con técnicas serológicas, destacando la aglutinación en suero (12 de 13 muestras) y sólo en uno (el último paciente diagnosticado en el año 2016) con técnica de ELISA, reflejando la introducción de nuevas técnicas diagnósticas validadas ${ }^{5,12}$.
En relación al diagnóstico serológico por aglutinación en suero en brucelosis, es importante aclarar que se considera clínicamente poco práctico esperar un alza en los títulos de anticuerpos por la demora que implica en iniciar el tratamiento antimicrobiano y se acepta como significativo para el diagnóstico un solo título $\geq 1: 80^{13}$.

Las ventajas de la técnica de ELISA versus aglutinación en suero, están en relación a una mayor sensibilidad en casos de brucelosis crónica, y a que es una técnica más rápida, menos engorrosa y que necesita menos horas hombre para su implementación ${ }^{5,14}$. Se estima que la detección simultánea de IgG e IgM presenta un mejor rendimiento diagnóstico; sin embargo, la detección de IgG por si sola sería más sensible que IgM y de mayor utilidad frente a las limitaciones de las técnicas de aglutinación.

Con respecto al tratamiento antimicrobiano utilizado en esta serie de pacientes, predominaron los esquemas asociados y prolongados, en concordancia con lo que sugiere la literatura médica ${ }^{15}$. El esquema más utilizado en nuestros pacientes, mientras permanecieron internados, fue doxiciclina-gentamicina, y la mayoría de ellos continuó con doxiciclina-rifampicina tras el alta hospitalaria por un total de seis semanas, obteniéndose curación en todos los pacientes. El tratamiento para brucelosis no complicada recomendado actualmente es usar doxiciclina asociada a estreptomicina, aunque puede ser reemplazado por gentamicina con resultados comparables e incluso superiores. El régimen doxiciclina-rifampicina presenta una tasa discretamente superior de fracaso de tratamiento, pero puede ser escogido al permitir la administración vía oral.

Deben completarse seis semanas de terapia antimicrobiana en total ${ }^{16}$. En los casos de brucelosis con focalización las terapias se prolongaron por cuatro meses (siendo lo mínimo recomendado tres meses) ${ }^{17}$, con respuesta satisfactoria en $100 \%$ de los casos.

\section{Limitaciones del estudio}

Esta serie cuenta con un tamaño de muestra pequeño (13 pacientes), lo que no permite establecer relaciones estadísticamente significativas entre las correlaciones halladas. Esto puede deberse en parte a que el análisis fue realizado sólo en tres centros de salud de la ciudad de Santiago, y a la baja incidencia de esta patología.

Junto a esto, la muestra fue conformada únicamente por pacientes que requirieron ingreso hospitalario, por lo que nuestro análisis podría subestimar la incidencia de esta patología y sobreestimar las complicaciones propias de la brucelosis.

Dado el diseño retrospectivo de nuestro estudio, en algunos casos nos encontramos con limitaciones del acceso a determinados datos, por falencias propias del registro que se realiza en la ficha clínica en la práctica médica habitual.

Por último, la ausencia de seguimiento sistemático, o 
al menos su reporte en ficha clínica, impidió recoger información acerca de eventuales recurrencias y/o recidivas.

Es necesario desarrollar nuevos estudios que permitan una detallada caracterización de la brucelosis en Chile además de conocer la verdadera prevalencia en la población general, como también en grupos de riesgo como veterinarios, ganaderos y personal de laboratorio que trabaja con muestras biológicas potencialmente contaminadas.

\section{Conclusiones}

La brucelosis es una zoonosis poco frecuente en el medio nacional, pero puede que exista un sub diagnóstico. En nuestra serie, correspondiente a una muestra de 13 pacientes, encontramos que se manifiesta como un cuadro inespecífico, tanto en la clínica como en el laboratorio, predominado el síndrome febril prolongado, por lo que la sospecha en base al antecedente del consumo de lácteos no pasteurizados o carne cruda o mal cocida resulta fundamental.

Estimamos que uno de los principales aportes de este trabajo es llamar la atención de los médicos clínicos para que consideren dentro del diagnóstico diferencial de la fiebre de origen desconocido y de las espondilodiscitis en Chile a la brucelosis como una eventual causa.

Es importante destacar que, en nuestro país, además de los métodos de diagnóstico recomendados clásicamente por la literatura médica, se han incorporado nuevos métodos de diagnóstico microbiológico validados internacionalmente, como la técnica de ELISA.

Pese a las limitaciones ya descritas, este corresponde al primer reporte de una serie clínica de pacientes adultos con brucelosis en un período de 16 años, por lo que es sin duda un avance en la construcción de la casuística nacional.

\section{Resumen}

Introducción: La brucelosis es una zoonosis provocada por Brucella spp, cuyo principal mecanismo de transmisión es mediante el consumo de lácteos no pasteurizados. Es de baja ocurrencia en Chile, por lo que los datos locales son escasos. Objetivo: Reportar y caracterizar la primera serie de casos clínicos de pacientes adultos con diagnóstico de brucelosis en Chile. Material y Método: Se describe una serie de 13 casos clínicos en pacientes diagnosticados entre el año 2000 y el 2016 en tres centros de la Región Metropolitana, Chile. Se realizó un análisis retrospectivo acerca de la presentación clínica, laboratorio, tratamiento antimicrobiano y morbimortalidad. Resultados: El promedio de edad fue 50 años. Ocho casos tenían antecedente de consumo de lácteos no pasteurizados. El motivo de consulta más frecuente fue sensación febril. El compromiso focal más frecuente fue el de columna vertebral. Sólo en un paciente se encontró hemocultivo positivo; en el resto el diagnóstico se hizo mediante técnicas serológicas. Los esquemas antimicrobianos más indicados fueron doxiciclina-rifampicina y doxiciclina-gentamicina. La estadía hospitalaria fue en promedio de 20 días. En todos los casos se logró curación clínica. Conclusiones: La brucelosis es una zoonosis infrecuente en Chile, produce un cuadro clínico inespecífico, por lo que se debe tener una alta sospecha para realizar el diagnóstico, basada en el antecedente del consumo de productos lácteos no pasteurizados o de carne mal cocida .

\section{Referencias bibliográficas}

1.- Dean A, Crump L, Greter H, et al. Clinical manifestations of human brucellosis: A systematic review and metaanalysis. PLoS Neglected Trop Dis 2012; 6: $1-10$.

2.- Martínez P. Brucelosis humana: situación epidemiológica en Chile, 2001-2010. Rev Chilena Infectol 2013; 30: 653-9.

3.- Seleem M, Boyle S, Sriranganathan N. Brucellosis: A re-emerging zoonosis. Vet Microbiol 2010; 140: 392-8.

4.- Gwida M, Al Dahouk S, Melzer F, Rösler U, Neubauer H, Tomaso H. Brucellosis-regionally emerging zoonotic disease. Croat Med J 2010; 51: 289-95.

5.- Aranís C, Oporto J, Espinoza M, Riedel I, Pérez C, García P. Utilidad de la determinación de anticuerpos IgG e IgM por ELISA e inmunocaptura en una serie clínica de brucelosis humana. Rev Chilena Infectol 2008; 25: 116-21.

6.- Kilic A, Metan G, Alp E. Clinical presentations and diagnosis of brucellosis. Recent Pat Antiinfect Drug Discov 2013; 8: 34-41

7.- Al Dahouk S, Tomaso H, Nöckler K, Neubauer H, Frangoulidis D. Laboratorybased diagnosis of brucellosis--a review of the literature. Part II: serological tests for brucellosis. Clin Lab 2003; 49: 577-89.

8.- Buzgan T, Karahocagil M, Irmak H, Baran A, Karsen H, Evirgen O, et al. Clinical manifestations and complications in 1028 cases of brucellosis: a retrospective evaluation and review of the literature. Int J Infect Dis 2010; 14: e469-78.

9.- Colmenero J, Reguera J, Martos F, Sánchez-de-Mora D, Delgado M, Causse M, et al. Complications associated with Brucella melitensis infection: A study with 530 cases. Medicine (Baltimore) 1996; 75: 195-211

10.- Reyes A, Villarroel J. Brucelosis en un escolar. Rev Chilena Infectol 2006; 23: 351-8.

11.- Franco M, Mulder M, Gilman R, Smits H. Human brucellosis. Lancet Infect Dis 2007; 7: 775-86.
12.- Araj G. Update on laboratory diagnosis of human brucellosis. Int J of Antimicrob Agents 2010; 36s: S12-7.

13.- Bosilkovski M. Clinical manifestations, diagnosis, and treatment of brucelosis. UpToDate 2016. Disponible en: http://www. uptodate.com/online. (Acceso el 27 de abril de 2017).

14.- Al Dahouk S, Nöckler K. Implications of laboratory diagnosis on brucellosis therapy. Expert Rev Anti Infect Ther 2011; 9 (7): 833-45.

15.- Yousefi-Nooraie R., Mortaz-Hejri S., Mehrani M., Sadeghipour P. Antibiotics for treating human brucellosis. Cochrane Database Syst Rev 2012; 10: CD007179.

16.- Solera J. Update on brucellosis: therapeutic challenges. Int J of Antimicrob Agents 2010; 36s: s18-s20.

17.- Colmenero J, Ruiz-Mesa J, Plata A, Bermúdez P, Martín-Rico P, Queipo-Ortuño M, et al. Clinical findings, therapeutic approach, and outcome of brucellar vertebral osteomyelitis. Clin Infect Dis 2008; 46: 426-33. 